\title{
Jejunal intussusception and polyps with different types of malignant transformation in Peutz-Jeghers syndrome: Report of a case
}

\author{
HONGKE CAI, WEI TIAN, MEIQI ZHOU, HAIFEI HE, YUE HU and YONGCHUAN DENG \\ Department of Surgical Oncology, Second Affiliated Hospital, Zhejiang University College of Medicine, \\ Hangzhou, Zhejiang 310009, P.R. China
}

Received July 24, 2012; Accepted October 12, 2012

DOI: $10.3892 / \mathrm{ol} .2012 .988$

\begin{abstract}
Intussusception and malignant polyps are complications of Peutz-Jeghers syndrome. Very few cases of intussusception combined with polyps with different types of malignant transformation in Peutz-Jeghers syndrome have been reported to date. In the present study, we describe a case of Peutz-Jeghers syndrome with jejunal intussusception and malignant hamartoma in the jejunum and descending colon, combined with mucinous adenocarcinoma in the sigmoid colon.
\end{abstract}

\section{Introduction}

Peutz-Jeghers syndrome is a rare autosomal dominant hereditary disease. It characterized by hyperpigmented macules and gastrointestinal polyps, the majority of which are hamartomas. It was once thought that in patients with Peutz-Jeghers syndrome there is a lower incidence of the transformation from hamartoma to malignance. However, increasing evidence indicates that these patients are not only at a higher risk of transformation to malignant polyps, but also of benign and malignant tumors in other organs throughout the body (1). Thus far, cases of intussusception and polyps occurring in different parts of the intestine and with different types of malignancy at the same time has rarely been reported.

\section{Case report}

A 38-year-old female presented at the outpatient department of the Second Affiliated Hospital of Zhejiang University College

Correspondence to: Professor Yongchuan Deng, Department of Surgical Oncology, Second Affiliated Hospital, Zhejiang University College of Medicine, 88 Jiefang road, Hangzhou, Zhejiang 310009, P.R. China

E-mail: dengyc001@hotmail.com

Key words: intussusception, hamartomatous polyp, malignant, Peutz-Jeghers syndrome of Medicine, China, with a history of recurrent abdominal pain of 10 years' duration and increased pain with vomiting for approximately 1 month. The patient described the pain as sudden-onset colic, and after diarrhea the feeling of pain would be relieved. A physical examination revealed hyperpigmented macules on her lips, buccal mucosa, the tips of fingers and toes, palms and soles of her feet (Fig. 1). The patient's father, her two younger brothers, one son and one daughter had experienced similar complaints (Fig. 2). Clear evidence of intestinal peristalsis in the left upper abdomen was observed when she experienced abdominal pain. Bowel sounds were increased. Laboratory investigations revealed normal hemoglobin, blood count and liver and renal function. Her erect abdominal radiograph did not reveal air-fluid levels. Breast ultrasonography showed fibroadenoma, and gynecological ultrasound showed ovarian cysts. E-colonoscopy showed multiple polyps within the colon, of which three were relatively large, in the transverse colon, descending colon and sigmoid colon. A diagnosis of Peutz-Jeghers syndrome was considered on the basis of clinical examination and the E-colonoscopy, and the patient underwent abdominal computed tomography (CT). Contrastenhanced abdominal CT showed that part of the intestine in the left upper abdomen with the surrounding mesenteric fat and vessels were invaginated into another section of the intestine (Fig. 3). A laparotomy revealed the part of the proximal jejunum, $20 \mathrm{~cm}$ away from the Treitz ligament, invaginated into the proximal jejunum, which determined the diagnosis of intussusception. A polyp was observed at the leading point of the intussusception. Following the removal of the intussusception, this polyp was completely resected (Fig. 4). The three larger polyps described previously were also completely resected. However, frozen section pathological diagnosis revealed hamartomas in the transverse colon, malignant hamartoma in the small intestine and descending colon, and mucinous adenocarcinoma infiltrating to the muscularis in the sigmoid colon (Fig. 5). Therefore, the method of surgery was altered to perform partial resection of the small intestine and left hemicolectomy. The patient was discharged 10 days after surgery. The histopathological examination following surgery supported the frozen section pathological diagnosis. Currently, the condition of the patient is stable and she remains in good health. Follow-up of the patient is ongoing. 

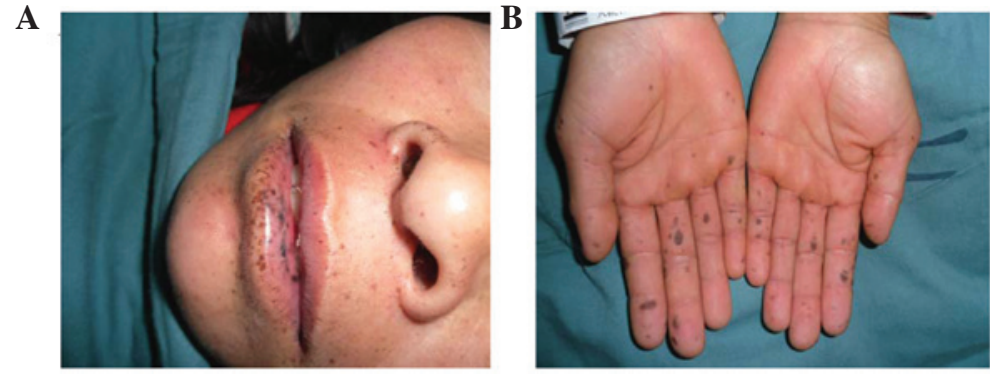

Figure 1. Images showing hyperpigmented macules on the patient's lips (A) and fingers (B).

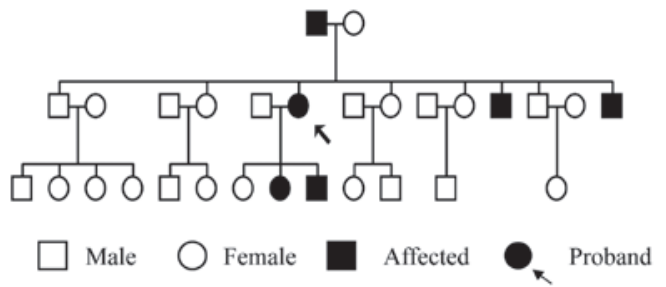

Figure 2. The pedigree revealed that the patient's father, two younger brothers, one son and one daughter had experienced similar complaints.

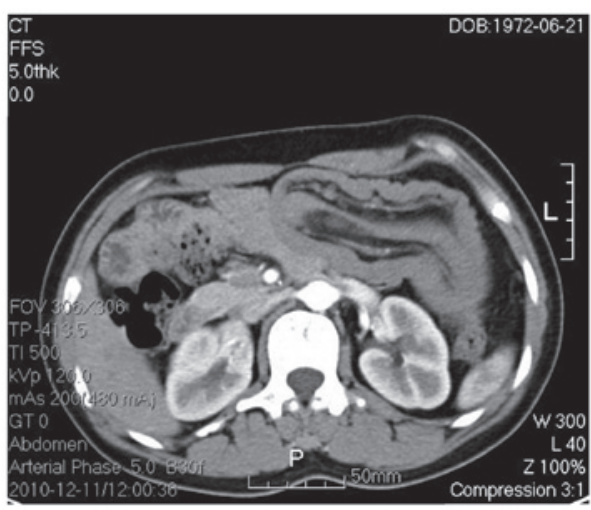

Figure 3. Contrast enhanced abdominal CT showing the intussusception.

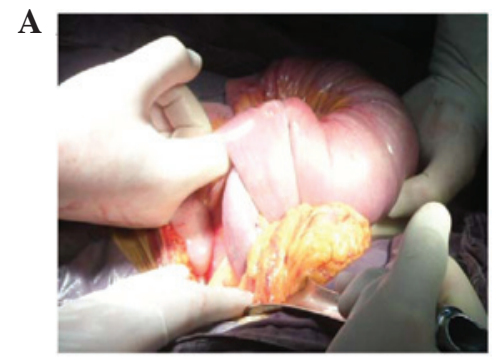

B

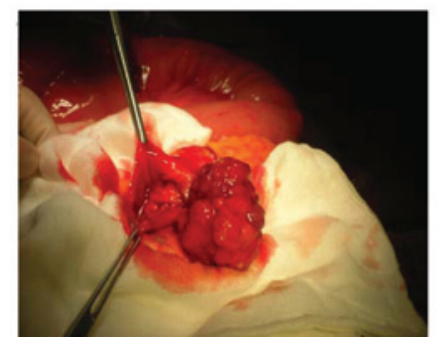

Figure 4. Surgical images. (A) The proximal jejunum intussusception (B) Polyp that acted as a lead point for the intussusception.

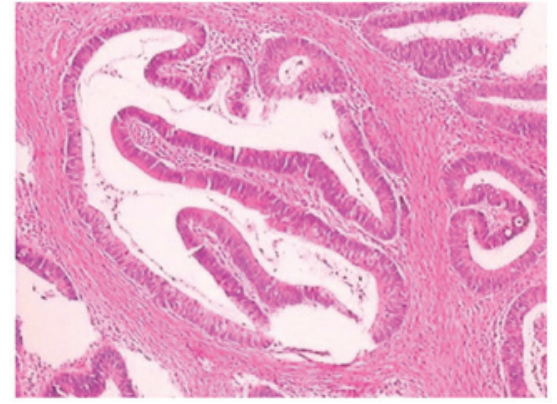

Figure 5. The histopathological examination revealed mucinous adenocarcinoma in the sigmoid colon.

\section{Discussion}

Peutz-Jeghers syndrome is a hereditary disease characterized as autosomal dominant. It was first described by Peutz (2) in 1921 and Jeghers et al (3) in 1949. The incidence has been estimated to be one in 200,000 births (4). Autosomal dominant inheritance, mucocutaneous pigmentation and hamartomatous polyps of the gastrointestinal tract are the three main features of Peutz-Jeghers syndrome. The most commonly affected sites of Peutz-Jeghers polyps in the gastrointestinal tract are the jejunum, colorectal region, duodenum and stomach, in decreasing frequency. The most common histological type of polyps is hamartoma, which demonstrates epithelial elements in the submucosa, muscularis propria and subserosa, and they frequently surround mucin-filled spaces (5). Peutz-Jeghers syndrome is a tumor susceptibility syndrome. The incidence of tumors in Peutz-Jeghers syndrome patients is 15 times higher than in normal individuals, and the incidence of malignant tumors is up to $20 \%$ (6). It is not only the hamartomas themselves that can evolve into adenomas and carcinomas, but also malignant tumors of other organs outside the gastrointestinal tract, occurring via de novo pathways (7). The cause of Peutz-Jeghers syndrome appears to be a germline mutation of the STK11/LKB1 (serine/threonine kinase 11) tumor suppressor gene in most cases $(8,9)$. In addition, the polyps with malignance may be associated with the interferon-induced transmembrane glycoprotein-1 (IFITM-1) (10), which may be a good marker to test for polyps in the process of malignant transformation in Peutz-Jeghers syndrome patients. The major therapeutic strategy used for Peutz-Jeghers syndrome is nonsteroidal anti-inflammatory drugs (NSAIDs). In addition, Wei et al (11) confirmed that rapamycin inhibited the enzyme encoded by STK11, which inhibited the growth of hamartoma. 
The indications for surgical treatments include patent polyps with abdominal cramps, intestinal bleeding, intussusception, intestinal obstruction and malignant polyps confirmed by endoscopic biopsy. The combination of surgical and endoscopic polypectomy is an efficient and practical choice in the management of polyps in patients with Peutz-Jeghers syndrome (12). Radical surgery is required only if the polyps are confirmed to be malignant. To date, cases of intestinal intussusception combined with multiple different types of malignant polyps have rarely been reported.

\section{References}

1. Niimi K, Tomoda H, Furusawa M, et al: Peutz-Jeghers syndrome associated with adenocarcinoma of the cecum and focal carcinoma in hamartomatous polyps of the colon: a case report. Jpn J Surg 21: 220-223, 1991.

2. Peutz JLA: Very remarkable case of familial polyposis of mucous membrane of intestinal tract and nasopharynx accompanied by peculiar pigmentations of skin and mucous membrane. Ned Tijdschr Geneeskd 10: 134-146, 1921 (In Dutch).

3. Jeghers H, McCusick VA and Katz KH: Generalized intestinal polyposis and melanin spots of the oral mucosa, lips and digits; a syndrome of diagnostic significance. N Engl J Med 241: 993-1005, 1949.
4. Chen HM and Fang JY: Genetics of the hamartomatous polyposis syndromes: a molecular review. Int J Colorectal Dis 24: 865-874, 2009.

5. Kakkar N, Vasishta RK, Poddar U and Thapa BR: Peutz-Jeghers polyposis with enteritis cystica profunda - a diagnostic pitfall. Pediatr Pathol Mol Med 22: 369-373, 2003.

6. Westerman AM, Entius MM, de Baar E, et al: Peutz-Jeghers syndrome: 78-year follow-up of the original family. Lancet 353: 1211-1215, 1999.

7. Taguchi T, Suita S, Taguchi S and Tanaka S: Peutz-Jeghers syndrome in children: high recurrence rate in short-term follow-up. Asian J Surg 26: 221-224, 2003.

8. Zubk KM and Eng C: Hamartomatous polyposis syndromes. Natl Clin Pract Gastroenterol Hepatol 4: 492-502, 2007.

9. Jamaludin AZ, Tlisinghe PU, Yapp SK and Chong VH: Solitary duodenal hamartomatous polyp with malignant transformation: report of a case. Surg Today 39: 527-532, 2009.

10. Ma Y, Zhang G, Fu X, et al: Wnt signaling may be activated in a subset of Peutz-Jeghers syndrome polyps closely correlating to LKB1 expression. Oncol Rep 23: 1569-1576, 2010.

11. Wei C, Amos CI, Zhang N, et al: Suppression of Peutz-Jeghers polyposis by targeting mammalian target of rapamycin signaling. Clin Cancer Res 14: 1167-1171, 2008.

12. Lin BC, Lien JM, Chen RJ, et al: Combined endoscopic and surgical treatment for the polyposis of Peutz-Jeghers syndrome. Surg Endosc 14: 1185-1187, 2000. 